\title{
A missense mutation in the bovine SLC35A3 gene, encoding a UDP-N-acetylglucosamine transporter, causes complex vertebral malformation
}

\author{
Bo Thomsen, ${ }^{1}$ Per Horn, ${ }^{1}$ Frank Panitz, ${ }^{1}$ Emøke Bendixen, ${ }^{2}$ Anette H. Petersen, ${ }^{1}$ \\ Lars-Erik Holm, ${ }^{1}$ Vivi H. Nielsen, ${ }^{1}$ Jørgen S. Agerholm, ${ }^{3}$ Jens Arnbjerg, ${ }^{4}$ and \\ Christian Bendixen ${ }^{1,5}$ \\ ${ }^{1}$ Department of Genetics and Biotechnology, and ${ }^{2}$ Department of Animal Health, Welfare and Nutrition, Danish Institute of \\ Agricultural Sciences, DK-8830 Tjele, Denmark; ${ }^{3}$ Department of Veterinary Pathobiology and ${ }^{4}$ Radiology, Royal Veterinary and \\ Agricultural University, DK-1870 Frederiksberg C, Denmark
}

\begin{abstract}
The extensive use of a limited number of elite bulls in cattle breeding can lead to rapid spread of recessively inherited disorders. A recent example is the globally distributed syndrome Complex Vertebral Malformation (CVM), which is characterized by misshapen and fused vertebrae around the cervico-thoracic junction. Here, we show that CVM is caused by a mutation in the Golgi-resident nucleotide-sugar transporter encoded by SLC35A3. Thus, the disease showed complete cosegregation with the mutation in a homozygous state, and proteome patterns indicated abnormal protein glycosylation in tissues of affected animals. In addition, a yeast mutant that is deficient in the transport of UDP-N-acetylglucosamine into its Golgi lumen can be rescued by the wild-type SLC35A3 gene, but not by the mutated gene. These results provide the first demonstration of a genetic disorder associated with a defective SLC35A3 gene, and reveal a new mechanism for malformation of the vertebral column caused by abnormal nucleotide-sugar transport into the Golgi apparatus.
\end{abstract}

[The sequence data from this study has been submitted to GenBank under accession no. AY160683. The following individuals kindly provided reagents, samples, or unpublished information as indicated in the paper: C.B. Hirschberg, C. Abeijon, and P.J. de Jong.]

CVM is a recessively inherited disorder with onset during fetal development, leading to frequent abortion of fetuses or perinatal death, and vertebral anomalies (Agerholm et al. 2001, 2004; Nielsen et al. 2003). Disease symptoms have not been observed in obligate carriers of CVM. The syndrome was first discovered in the Danish Holstein population in 1999, but shortly thereafter reports documented the presence of CVM in the United States (Duncan et al. 2001), the United Kingdom (Revell 2001), the Netherlands (Wouda et al. 2000), and in Japan (Nagahata et al. 2002). Genealogical records allowed us to trace the origin of the disease-causing allele to a common ancestral bull, Carlin-M Ivanhoe Bell, which has been used in dairy cattle breeding worldwide for two decades due to the superior lactation performance of his daughters. Consequently, the number of animals genetically related to the founder bull is very high, and the disease-causing mutation is now prevalent among Holstein cattle throughout the world. For example, the frequencies of CVM carriers among top sires were 31\% in Denmark in 1999 (A.H. Petersen, unpubl.) and $32.5 \%$ in Japan in 2001 (Nagahata et al. 2002). Clearly, the use of relatively few elite bulls can advance productivity in a breed; yet, this strategy also greatly augments the risk of increasing the frequency of deleterious recessive mutations.

\footnotetext{
${ }^{5}$ Corresponding author.
}

E-mail christian.bendixen@agrsci.dk; fax 45-89991300.

Article published online ahead of print. Article and publication date are at http://www.genome.org/cgi/doi/10.1101/gr.3690506. Freely available online through the Genome Research Immediate Open Access option.
Detailed clinical characterization of CVM demonstrated a composite phenotype with axial skeletal deformities such as hemivertebrae, misshaped vertebrae, ankylosis of mainly the cervico-thoracic vertebrae, scoliosis, and symmetric arthrogryposis of the lower limb joints, craniofacial dysmorphism, as well as cardiac anomalies (Agerholm et al. 2001, 2004; Nielsen et al. 2003) (Fig. 1A,B). The diagnosis of CVM can be difficult to make because of significant clinical heterogeneity among affected calves and the occurrence of phenocopies. A presumptive diagnosis can usually be obtained at necropsy in combination with pedigree information; however, a definitive diagnosis requires DNA testing (Agerholm et al. 2004). The vertebrate axial skeleton is derived from somites generated by segmentation of the paraxial mesoderm in an intricate process that requires participation of two signal-transduction pathways, Wnt and Notch (Pourquie 2003; Aulehla and Herrmann 2004). Thus, somitogenesis involves a molecular oscillator mechanism consisting of cyclic expression of genes encoding several Notch pathway components such as the glycosyltransferase Lunatic Fringe, Notch ligands, downstream target genes of Notch as well as the Wnt pathway regulator Axin2. Moreover, morphogen gradients of Fgf8 (Dubrulle and Pourquie 2004) and Wnt3a (Aulehla et al. 2003) in the presomitic mesoderm are crucial for determining the position of somitic boundaries. Furthermore, somites differentiate into vertebrae, each having a unique morphological identity that depends on its position along the body axis. The axial identity of the vertebrae is specified by expression of an appropriate set of 

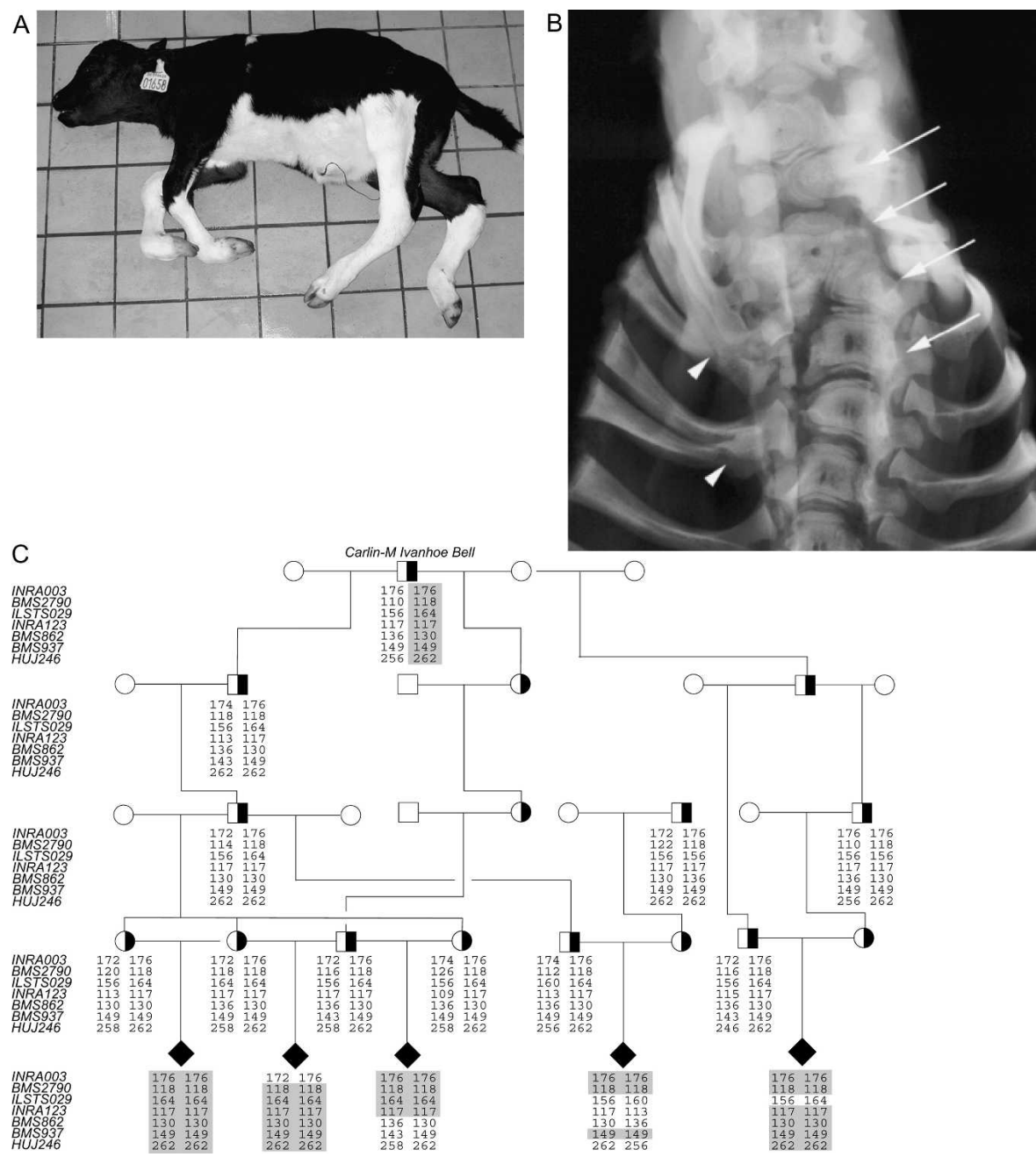

Figure 1. Complex vertebral malformation. (A) Affected calf. Notice the short neck and contraction of the distal joints. (B) Radiograph of the cervico-thoracic part of the vertebral column (arch removed), showing multiple malformed vertebrae and scoliosis (arrows) and fusion of the proximal part of several ribs (arrowheads), ventro-dorsal projection. (C) Pedigree and haplotype analysis. All affected calves can be traced back to a common ancestor Carlin-M Ivanhoe Bell. Genotypes of seven polymorphic makers at the disease locus on BTA3 that helped to define the disease locus are shown. The combined genotyping results suggested that the disease gene is located near BMS2790 in an $\sim 5 \mathrm{cM}$ region flanked by the markers INRA003 and ILSTS029. The shared disease-associated haplotype is shaded in gray. (Filled symbols) Affected; (open symbols) unaffected; (half-filled symbols) carrier.

$H O X$ genes in a coordinated fashion with the segmentation oscillator (Dubrulle et al. 2001; Zakany et al. 2001).

The purpose of the present study was to identify the mutation responsible for CVM. The data demonstrate that the disease gene encodes a member of the solute carrier family SLC35, which are enzymes transporting nucleotide-sugars from the cytosol into the lumen of the endoplasmic reticulum and/or the Golgi apparatus (Ishida and Kawakita 2004). In these organelles, nucleotidesugars are utilized by glycosyltranferases to synthesize the sugar chains of glycoproteins, glycolipids, and carbohydrate polymers. Several studies lend support for an essential role of nucleotidesugar transportation in development and disease. Thus, in Drosophila, impaired function of Fringe connection (encoded by frc), a transporter with multisubstrate specificity, was shown to have detrimental effects on fibroblast growth factor, Hedgehog, Wingless-dependent signalling, as well as to disrupt the Notch path- way (Goto et al. 2001; Selva et al. 2001). Two Caenorhabditis elegans nucleotidesugar transporters with mutant phenotypes have been characterized. Thus, the C. elegans sqv-7 mutant, which is defective in a homolog of frc, exhibits severe defects in vulval invagination and impairment of embryonic development, presumably reflecting an inability to transport nucleotide-sugar substrates required for glycosaminoglycan biosynthesis into the Golgi (Bulik et al. 2000; Berninsone et al. 2001; Hwang and Horvitz 2002). Furthermore, mutation of the UDP-galactose and UDP- $\mathrm{N}$-acetylglucosamine transporter encoded by srf-3 in C. elegans confers resistance to bacterial infections due to failure of bacteria to adhere to the altered glycoconjugate composition of the mutant nematode cuticle (Hoflich et al. 2004). Observations on human glycosylation diseases further attest to the importance of nucleotide-sugar transportation. Thus, the human disease called congenital disorder of glycosylation (CDG) type IIc (formerly leukocyte adhesion deficiency type II) occurs when GDP-fucose uptake into the Golgi is defective, resulting in clinical manifestations such as facial dysmorphology, growth and mental retardation, and lack of cell-surface expression of fucosylated glycoconjugates like the $\mathrm{ABH}$ and Lewis-related blood group antigens. Also, fucose-containing carbohydrate ligands required for selectin interactions are absent, leading to leukocyte adhesion deficiency (Lubke et al. 2001; Luhn et al. 2001). Recently, a related glycosylation disorder CDG type IIf was shown to result from inactivation of the Golgi CMP-sialic acid transporter (Martinez-Duncker et al. 2005).

Bovine SLC35A3 is the first nucleotide-sugar transporter shown to play a role in the development of the axial skeleton, demonstrating that some of the molecular mechanisms that operate during formation of vertebrae and ribs depend on carbohydrate modification in the Golgi apparatus. The present disease model may be exploited to predict the human clinical phenotype as well as to provide further insight into the disease mechanisms.

\section{Results}

\section{Genetic mapping of the disease gene}

Affected animals are expected to be homozygous in polymorphic markers flanking the disease locus, as all chromosomes carrying the causative mutation descended from a common founder bull (Agerholm et al. 2001). Therefore, we performed a genome-wide scan for regions identical-by-descent. Seven calves diagnosed 
with CVM based on post-mortem examination as described (Agerholm et al. 2001) plus their parents were used in the initial genome scan using a panel of 194 microsatellite markers covering all 29 autosomes with pairwise distances between 10 and $20 \mathrm{cM}$. The results revealed that all seven calves were homozygous for the same allele of marker INRAO03 $(59.4 \mathrm{cM})$, whereas five calves had identical alleles in an adjacent marker HUJ246 $(67.9 \mathrm{cM})$ on BTA3. To confirm this observation and to more accurately define the disease locus, we expanded the analysis with additional affected calves sampled by the Danish surveillance program for genetic defects to include a total of 30 affected calves plus available parental animals, which were genotyped with an increased marker density in the interval between INRAOO3 and HUJ246 (Fig. 1C). The evidence for linkage was highly significant with twopoint lod scores of $8.13(\theta=0.00)$ for BMS2790 (62.4 $\mathrm{cM}), 7.53(\theta=0.00)$ for INRAOO3 $(59.4 \mathrm{cM})$, and 5.12 $(\theta=0.04)$ for ILSTSO29 $(64.9 \mathrm{cM})$. Haplotype analysis of the five-generation pedigree shown in Figure 1C revealed several critical recombinants that allowed us to narrow down the position of the disease gene. Thus, all affected calves were homozygous in BMS2790, and recombination between BMS2790 and the nearby markers INRAOO3 and ILSTSO29 defined the limits of the CVM disease locus.

The cattle genome is only sparsely annotated with known genes, so in order to identify positional candidate genes we anchored the disease locus to the human genome by screening a bovine large-insert bacterial artificial chromosome (BAC) library for clones containing either one of the three markers INRA003, BMS2790, or ILSTSO29. BAC end sequences were subsequently compared with the human genome sequence by BLAST searches, which showed that the INRA003-BMS2790-ILST029 interval corresponds to an $\sim 5-6 \mathrm{Mb}$ region in HSA1p21.2-21.3 (Fig. 2A).

\section{Candidate gene selection}

The bovine-human comparative gene map of the disease locus contains $\sim 20$ known or predicted genes, none of which has previously been associated with defects in vertebral column formation. However, a rationale for selection of a candidate gene was provided by the notion that similar disease phenotypes can arise from mutations in different genes engaged in the same signaling cascade. Thus, malfunction of Notch-signaling genes, like the CVM disease gene, causes complex phenotypes, including vertebral and costal deformations as well as abnormalities in various nonskeletal tissues and organs (Conlon et al. 1995; Li et al. 1997; Oda et al. 1997; Evrard et al. 1998; Jiang et al. 1998; Kusumi et al. 1998; Zhang and Gridley 1998; Hamada et al. 1999; Bulman et al. 2000). The Notch receptors and their ligands, Jagged and Delta, are cell-surface transmembrane proteins, which serve to transduce signals between neighboring cells. Significantly, this cellto-cell communication is controlled by the Golgi-resident glycosyltransferase Fringe, which modulates the ability of the ligands to activate the Notch receptors by adding $\mathrm{N}$-acetylglucosamine onto fucose residues attached to Notch (Hicks et al. 2000). In this scenario, it is particularly intriguing that the disease locus harbors the nucleotide-sugar transporter SLC35A3 (solute carrier family 35 , member A3). The SLC35A3 gene is ubiquitously expressed and the encoded transporter is localized in the Golgi apparatus (Ishida et al. 1999). Furthermore, determination of its substrate specificity using Golgi-enriched fractions of yeast cells expressing either the human SLC35A3 gene (Ishida et al. 1999) or the canine counterpart (Guillen et al. 1998) showed efficient transport of UDP-N-acetylglucosamine into the Golgi lumen, but not of UDP-galactose or CMP-sialic acid. We hypothesized that one effect of a mutation in SLC $35 A 3$ would be to deprive Fringe of its sugar donor, which in turn would affect the glycosylation status of Notch.

First, we sought to verify that $S L C 35 A 3$ in fact is positioned at the disease locus by building a BAC contig consisting of 22 individual clones between BMS2790 and INRAOO3 (Fig. 2A). Eight bovine homologs of human genes known to be located in HSA1p21.2, including SLC35A3, were successfully amplified by PCR using the BAC contig clones as templates. This result suggested that gene content and order in the CVM region and HSA1p21.2 were highly conserved between the two species.

Secondly, we examined whether supportive evidence for a causative role of $S L C 35 A 3$ in CVM can be observed at the protein level. Thus, glycosylation inside the Golgi apparatus modifies a wide range of proteins, suggesting that a deficiency in Golgi nucleotide-sugar transportation should be detectable by twodimensional protein gel analysis. Indeed, 2DE analyses of cardiac 
and skeletal muscle tissues revealed a series of protein spots with characteristic shifts in both molecular weight and charge consistent with changes in glycosylation (Fig. 2B). To investigate this in greater detail, we used MALDI-TOF mass spectrometry to identify the prominent protein pattern (encircled) that showed both altered migration as well as accumulation in the tissues of affected animals. All spots in the encircled patterns were identified as $\alpha 1$-antitrypsin, which is a serine proteinase inhibitor with a major role in protection of lung tissue from proteolytic destruction by elastase (Carrell and Lomas 2002). $\alpha 1$-antitrypsin is a glycoprotein modified by asparagine (N)-linked glycans, which assist in folding, quality control, and intracellular transport of the protein (Cabral et al. 2001). Several mutant forms of $\alpha 1$-antitrypsin are incapable of correct folding, which leads to protein accumulation in hepatocytes and a cytotoxic response in the form of cirrhosis of the liver or hepatocellular carcinoma. Another consequence of the incorrectly folded $\alpha 1$-antitrypsin is uncontrolled elastase activity, which results in pulmonary emphysema typically in the third or fourth decade of life (Crowther et al. 2004). Moreover, $\alpha 1$-antitrypsin is underglycosylated in patients with congenital disorder of glycosylation (CDG) type Ia and type Ic, both of which are deficient in the formation of the common dolichol-linked oligosaccharide precursor of N-glycans (Mills et al. 2001, 2003). Biosynthesis of N-glycans involves attachment of $\mathrm{N}$-acetylglucosamine in the Golgi apparatus, and therefore, it can be expected that a deficiency in UDP- $N$-acetylglucosamine uptake will affect glycosylation of $\alpha 1$-antitrypsin as observed. Taken together, these data therefore support our assumption that SLC35A3 is mutated in CVM.

\section{Mutation detection}

Using shotgun sequencing of BAC DNA, we determined the complete 22,400-bp sequence of the bovine SLC35A3 gene, and established the exon-intron organization by alignment with the full-length SLC $35 A 3$ cDNA sequence. Conceptual translation of the open reading frame predicted a protein of 326 amino acids with a highly conserved sequence, showing $98 \%$ identity to the human and canine SLC35A3 transporters, and 96\% and 83\% sequence identity to the mouse and frog SLC35A3 transporters, respectively (Fig. 3A). To identify the putative mutation in SLC 35A3, we compared the cDNA sequences of unaffected and affected calves, which uncovered a transversion ( $G$ to $T$ ) that replaces valine at position 180 with phenylalanine (Fig. 3B). No other mutations were detected. Moreover, point mutations in coding regions of several genes have been recognized to influence gene function by affecting the splicing pattern (Cartegni et al. 2002) We investigated this possibility by Northern blot analysis of SLC35A3 gene expression (Fig. 3C). The result revealed two large mRNA transcripts in both unaffected and CVM calves, which is consistent with the observed transcript sizes of $6.2 \mathrm{~kb}$ and $2.4 \mathrm{~kb}$ in various human tissues (Ishida et al. 1999), and there was no apparent effect of the mutation on splicing accuracy or efficiency. Subsequent large-scale genotyping strongly advocated that the V180F substitution is pathogenic. Thus, all calves diagnosed as CVM affected were homozygous with respect to phenylalanine, whereas 108 verified carriers of CVM were heterozygous. Also, we genotyped more than 1500 phenotypically normal animals descending from Carlin-M Ivanhoe Bell, and none
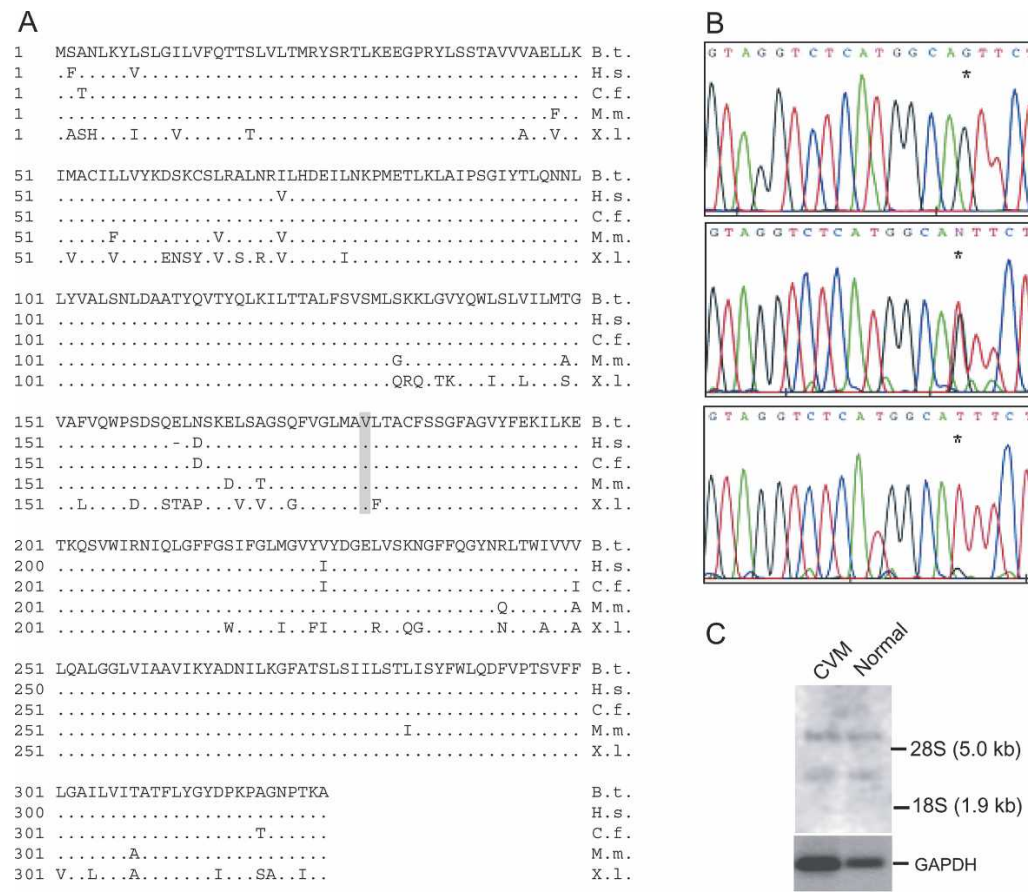

Figure 3. Mutation detection in SLC35A3. (A) The deduced amino acid sequence of bovine SLC $35 A 3$ and comparison with its homologs in human (Homo sapiens; BAA77841), dog (Canis familiaris; AAC39260), mouse (Mus musculus; AAH24110), and frog (Xenopus laevis; CAD47803). Dots indicate residues that match the Bos taurus sequence. Dashes indicate gaps that have been introduced to optimize the alignment. The valine at position 180 substituted by phenylalanine in CVM is indicated in bold type and shaded in gray. (B) Electropherograms showing the nucleotide sequences across the $\mathrm{G} \rightarrow$ T mutation (indicated by an asterix) in normal $(+/+)$, carrier $(+/-)$, and CVM-affected $(-/-)$ animals. (C) Northern blot analysis of SLC35A3 mRNA. Poly $(\mathrm{A})^{+}$RNA was isolated from kidney tissue of CVM calves and unaffected calves and analyzed by hybridizing Northern blots with a ${ }^{32} \mathrm{P}-$-labeled SLC35A3 probe. Subsequently, the membranes were stripped and reprobed with a ${ }^{32}$ P-labeled GAPDH (glyceraldehyde-3-phosphate dehydrogenase) fragment.

\section{Genome Research}

www.genome.org 
were homozygous for phenylalanine. Furthermore, analysis of more than 500 animals of the Holstein breed selected as being unrelated to the founder and $\sim 300$ animals of 12 other breeds did not identify any animals with phenylalanine at position 180 . These data, taken together with the fact that valine at position 180 is evolutionarily conserved, support the conclusion that the $\mathrm{G}$ to $\mathrm{T}$ transversion in SLC35A3 is the disease-causing mutation.

\section{Functional testing of the mutation in SLC35A3}

Next, we used a yeast complementation assay (Abeijon et al. 1996) to evaluate the significance of the V180F substitution (Fig. 4A). The Kluyveromyces lactis mutant mnn2-2, which is deficient in transport of UDP- $N$-acetylglucosamine into Golgi vesicles, lacks terminal $\mathrm{N}$-acetylglucosamine in its mannan chains of mannoproteins on the cell surface. It has been demonstrated previously that this biochemical defect of the mnn2-2 mutant can be rescued by expression of the mammalian SLC35A3 gene but not by other nucleotide-sugar transporters, for example, that

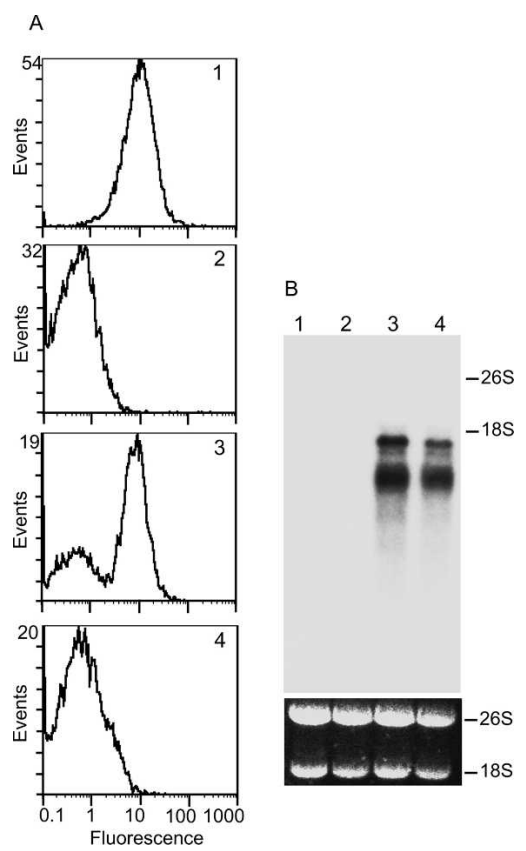

Figure 4. Analysis of $K$. lactis cell-surface labeling by flow cytometry. $(A)$ The $K$. lactis mutant mnn2-2 lacks terminal $\mathrm{N}$-acetylglucosamine in its cell surface glycoproteins. Wild-type (MG1/2) and mnn2-2 $\left(\mathrm{KL}_{3}\right)$ cells can therefore be differentially labeled by incubation with FITC-conjugated wheat-germ agglutinin (WGA), which has an affinity for $\mathrm{N}$ acetylglucosaminyl residues. Phenotypic correction of the cell-surface defect was observed only in mnn2-2 transformants expressing wild-type SLC35A3. (Panel 1) Wild-type MG1/2 transformed with empty pE4 vector; (Panel 2) mutant mnn2-2 transformed with empty pE4 vector; (Panel 3) mutant mnn2-2 expressing the wild-type SLC35A3 gene; (Panel 4) mutant mnn2-2 expressing the mutated SLC35A3 gene with the V180F substitution. $(B)$ Northern blot analysis. Total RNA extracted from the transformed yeast cells was separated by gel electrophoresis, blotted onto nylon membranes, and hybridized with a radioactively labeled fulllength SLC35A3 CDNA probe. Positions of the $26 \mathrm{~S}$ and $18 \mathrm{~S}$ ribosomal RNA are indicated. (Lane 1) Wild-type MG1/2 transformed with empty $\mathrm{pE} 4$ vector; (lane 2) mutant mnn2-2 transformed with empty $\mathrm{pE} 4$ vector; (lane 3) mutant mnn2-2 expressing the wild-type SLC35A3 gene; (lane 4) mutant mnn2-2 expressing the mutated SLC35A3 gene with the V180F substitution. Ethidium bromide stained $26 \mathrm{~S}$ and $18 \mathrm{~S}$ rRNA to control for equal loading is shown below the Northern blot. of UDP-galactose (Guillen et al. 1998). In agreement with previous data, we found that the mnn2-2 mutant was fully complemented by expression of the bovine wild-type $S L C 35 A 3$ gene. In contrast, transformation with the mutated SLC $35 A 3$ gene did not increase surface expression of $\mathrm{N}$-acetylglucosamine relative to that obtained in mnn2-2 cells with the empty vector. Thus, the absence of phenotypic correction shows that the V180F replacement in SLC35A3 strongly affects nucleotide-sugar transportation, although the yeast complementation assay does not allow us to assess whether activity is partially or completely lost. To assess the level of gene expression in the transformed yeast cells, we used Northern blot analysis with a probe specific for the SLC35A3 transcript. Essentially identical profiles with two major transcripts were observed, demonstrating that the wild-type and the mutated SLC35A3 gene were equally expressed in the mnn2-2 yeast mutant (Fig. 4B). Altogether, these results imply that the missense mutation in $S L C 35 A 3$ is responsible for CVM, and also excludes the possibility that a mutation in another gene tightly linked to $S L C 35 A 3$ is causing the defect.

\section{Discussion}

Intense breeding programs often involve mating of descendants of a particular bull, thereby increasing the familial relationship in the cattle population. By the time it was realized that Carlin-M Ivanhoe Bell carries a lethal mutation, the widespread use of this elite bull had already increased carrier frequencies of CVM in dairy cattle to an alarming $20 \%-30 \%$ in many countries. This prompted a search for the disease gene, which we now have identified as the SLC $35 A 3$ gene encoding a Golgi-resident transporter of UDP- $\mathrm{N}$-acetylglucosamine. Remarkably, Carlin-M Ivanhoe Bell was also a carrier of another disease called bovine leukocyte adhesion deficiency (BLAD), which is an immunodeficiency disorder caused by a recessive mutation in the gene encoding the leukocyte $\beta 2$ integrin subunit CD18 (Shuster et al. 1992) on BTA1 (Rexroad et al. 1999). A decade of DNA testing has reduced the frequency of the pathogenic allele and today the disease is efficiently controlled. The two defective genes responsible for CVM and BLAD are located on different chromosomes and therefore inherited independently. Genetic testing has shown that Carlin-M Ivanhoe Bell received both disease-causing mutations from his sire Pennstate Ivanhoe Star, whereas his grandsire Osborndale Ivanhoe is a BLAD carrier, but free of CVM. This suggests that the CVM mutation was either generated in Pennstate Ivanhoe Star or that it originates from the maternal side. With the identification of the genetic basis of the CVM disease, further transmission of the mutation can now be prevented through DNA testing of animals to be used for breeding.

Haploinsufficiency for the SLC35A3 transporter appears to be tolerated because heterozygous carriers are asymptomatic, whereas in its homozygous state, the mutation typically causes death in utero. Yet some fetuses do develop to term, although with severe defects. This might suggest that the mutated transporter retains some residual activity, occasionally allowing fetal development past critical stages, or alternatively, that the lack of SLC35A3 is alleviated by partial functional compensation by another nucleotide-sugar transporter with the ability to transport UDP-N-acetylglucosamine into the Golgi lumen. Thus, SLC35D2/HFRC1, and possibly also SLC35D1 (alias UGTrel7), have transporting activity toward a range of nucleotide-sugar substrates including UDP- $N$-acetylglucosamine (Muraoka et al. 
2001; Suda et al. 2004; Ishida et al. 2005). It is significant, however, that only SLC35D2/HFRC1 is localized in the Golgi apparatus like SLC35A3, while SLC35D1 is found in the endoplasmic reticulum. These nucleotide-sugar transporters exhibit $\sim 50 \%$ similarity with Drosophila Fringe connection and C. elegans SQV7 , which has been taken to indicate that they may serve corresponding roles in vertebrates (Goto et al. 2001; Selva et al. 2001; Suda et al. 2004; Ishida et al. 2005). Their cellular functions are at present unknown, and it awaits further work to clarify whether SLC35D2/HFRC1 or SLC35D1 interacts with SLC35A3 to regulate the formation of the vertebral column or whether they function in independent glycosylation pathways.

It is as yet undefined which specific molecular and cellular functions are affected by loss of UDP- $N$-acetylglucosamine transportation in CVM, but the composite disease phenotype with defects in multiple tissues suggest that several pathways and biochemical mechanisms are impaired. Proteomic changes were detected in the tissues of affected calves, and biochemical characterization of these proteins will help to understand the complexity of the phenotype. For example, we identified a series of $\alpha 1$ antitrypsin isoforms with altered mass and charge accumulating in the muscle tissue of affected animals, which indicates that aberrant asparagine (N)-linked glycosylation is associated with CVM. N-glycans are ubiquitous and important in many biological processes, which is reflected in the high number of human genetic diseases that are caused by mutations in the $\mathrm{N}$-glycan biosynthetic and secretory pathway (Marquardt and Freeze 2001). Conceivably, the Golgi-localized glycosyltransferases that use UDP- $N$-acetylglucosamine to synthesize $\mathrm{N}$-linked glycans are potentially affected by a mutation in $S L C 35 A 3$. Thus, it is noteworthy that mice lacking Mgat1 encoding $\mathrm{N}$-acetylglucosaminyltransferase I die before birth with developmental defects in somite formation and neural tissue morphogenesis due to the absence of hybrid and complex N-linked carbohydrates (Ioffe and Stanley 1994). Similarly, mutations in Mgat2, which catalyzes the formation of complex N-glycans, are associated with severe abnormalities in both mice and humans (responsible for CDG type IIa), which are characterized by craniofacial dysmorphism, thoracolumbar kyphoscoliosis, and heart defects (Wang et al. 2001).

The V180F substitution may also affect the antiporter function of SLC35A3. Thus, after uptake of nucleotide-sugars in the Golgi, the sugar moiety is transferred to various acceptors by glycosyltransferases, whereas the nucleoside diphosphate is hydrolyzed into nucleoside monophosphates by nucleoside diphosphatases. The latter reaction is crucial because nucleoside diphosphates are potent inhibitors of glycosyltransferases. Moreover, the nucleoside monophosphates are required by the nucleotidesugar transporters, which operate as antiporters by coupling nucleotide-sugar transport into the Golgi with an equimolar export of the corresponding nucleoside monophosphate. Loss or impairment of nucleoside diphosphatases has consequences for the function of the antiport cycle and glycosylation. For example, mutants lacking the GDPase gene GDA1 in Saccharomyces cerevisiae or $K$. lactis showed altered glycosylation of proteins and lipids due to reduced transport of GDP-mannose (and UDP- $N$ acetylglucosamine in $K$. lactis) into Golgi vesicles as well as inhibition of glycosyltransferases (Abeijon et al. 1993; Berninsone et al. 1994; Lopez-Avalos et al. 2001). Similarly, mutation of the mig-23 nucleotide diphosphatase in C. elegans has been shown to affect gonad morphology due to aberrant glycosylation of the MIG-17 protease (Nishiwaki et al. 2004).

Signal transduction relying on glycosylation is likely to be affected in CVM, and an attractive model is that obstruction of the Notch pathway is a contributing factor. An argument in favor of this proposal is the phenotypic similarity between the CVM defects and diseases such as those caused by defective Notch ligands JAGGED1 in Alagille syndrome (Li et al. 1997; Oda et al. 1997) and DELTA-LIKE 3 in spondylocostal dysostosis (Bulman et al. 2000) in humans, or the lunatic fringe-deficient mouse model (Evrard et al. 1998; Zhang and Gridley 1998). The underlying biochemical basis may be that regulation of Notch signaling relies on covalent attachment of $\mathrm{N}$-acetylglucosamine to $\mathrm{O}$-linked fucose on the Notch receptors by the glycosyltransferase activity of Fringe. Importantly, Fringe has strong specificity for fucose as acceptor and for UDP- $\mathrm{N}$-acetylglucosamine as donor and shows no activity with other donor-acceptor combinations (Bruckner et al. 2000; Moloney et al. 2000). UDP- $\mathrm{N}$-acetylglucosamine is synthesized in the cytosol and must be actively translocated across the Golgi membrane before it can be utilized by Fringe (Abeijon et al. 1997). Our data suggest that SLC35A3 provides this necessary link, thereby being involved in controlling the subset of Notch functions that depend upon $\mathrm{N}$-acetylglucosamine modification. Precedence for such a mechanism comes from analysis of Drosophila mutants lacking Fringe connection, showing that the Notch pathway is disrupted at two levels. First, Fringe-mediated attachment of sugar residues to Notch was reduced, and secondly, the level of proteolytic maturation of the Notch receptor was significantly lowered, probably as a result of abnormal Nlinked glycosylation (Goto et al. 2001; Selva et al. 2001).

Mutations affecting the function of glycosaminoglycans or proteoglycans have been implicated in numerous human and mouse pathologies (Princivalle and de Agostini 2002). Importantly, biosynthesis of glycosaminoglycans in the Golgi has been shown to be affected by defective nucleotide-sugar transporters. Thus, a Madin-Darby canine kidney cell line with strongly reduced UDP-galactose transportation was, in contrast to the parental cell line, unable to produce keratan sulphate, the only glycosaminoglycan containing galactose in its carbohydrate polymer (Toma et al. 1996). Also, C. elegans development requires glycosaminoglycans, and the defects displayed by $s q v-7$ mutants are probably a result of reduced synthesis of chondroitin and heparan sulphate (Bulik et al. 2000; Berninsone et al. 2001; Hwang and Horvitz 2002). Glycosaminoglycans modulate signaling mediated by Hedgehog, Wingless/Wnt, and FGF, and the disrupted signaling in these pathways and the associated phenotypes, which are observed in mutants of Fringe connection, can be accounted for by lack of heparan sulphate synthesis (Goto et al. 2001; Selva et al. 2001). These findings may be relevant to the understanding of the CVM phenotype, because heparan sulphate, keratan sulphate, and hyaluronic acid all contain $\mathrm{N}$ acetylglucosamine residues, making it plausible that their biosynthesis and biological activity could be affected by the mutation in SLC 35A3.

\section{Methods}

\section{Genome-wide scan}

PCR reactions were analyzed on an ABI 377 Sequencer (Applied Biosystems) and alleles were assigned with the Genotyper program, version 2.1. Two-point linkage analysis was performed using CRIMAP 2.4 (Lander and Green 1987) in a pedigree comprising 85 individuals, of which 29 were affected calves. The bull KOL Nixon sired 26 of these calves and 21 shared T. Burma as the

\section{Genome Research}

www.genome.org 
maternal grandsire. Both bulls have familial relationships with Carlin-M Ivanhoe Bell. Three different bulls also descending from Carlin-M Ivanhoe Bell sired the remaining calves.

\section{Genomic and cDNA sequences of SLC35A3}

A bovine BAC clone containing the SLC35A3 gene was mechanically sheared and size-selected fragments (500-1000 bp) were subcloned into TOPOShotgun Subcloning Kit (Invitrogen). Approximately 2000 clones were sequenced on a MegaBACE1000 (Amersham Pharmacia Biotech) using DYEnamic ET Dye Terminator Cycle Sequencing Kit (Amersham Pharmacia Biotech). The sequencing data were assembled into several contigs using the PHRED/PHRAP software (http://www.phrap.org). Gap closure between contigs was established by primer extension on BAC DNA using BigDye Terminator Cycle Sequencing Kit (PE Applied Biosystems). The complete genomic SLC35A3 sequence spanning $\sim 22,400$ bp (GenBank accession no. AY160683) was assembled with Sequencher (Version 4.0.5, Gene Codes Corporation). Total RNA was isolated (RNA Isolation Kit, Stratagene) from heart and muscle tissues from both unaffected and affected calves and used for first-strand cDNA synthesis with oligo- $\left(\mathrm{dT}_{12-18}\right)$ and Superscript II (Invitrogen). Subsequently, the SLC35A3 coding region was amplified by PCR using primer pairs designed on the basis of sequence homology between the human and canine genes, GenBank accession numbers AB021981 and AF057365, respectively.

\section{Northern blot analysis}

Total RNA was prepared from various tissues or yeast cells following the Qiagen RNeasy protocol as recommended by the manufacturer. Poly (A) ${ }^{+}$RNA was isolated using the Dynabeads mRNA DIRECT Kit (Dynal Biotech) following the instructions of the manufacturer. Approximately $10 \mu \mathrm{g}$ of total RNA or $0.6 \mu \mathrm{g}$ of poly $(\mathrm{A})^{+}$RNA was separated on a $1 \%$ agarose-glyoxal gel and subsequently transferred to positively charged nylon membranes using the NorthernMax-Gly kit (Ambion) according to the supplier's instructions. The probes were radioactively labeled with the Nick Translation System (GIBCO BRL) using $\left[\alpha-{ }^{32} \mathrm{P}\right] \mathrm{dCTP}$ from Amersham Biosciences.

\section{Proteome analysis}

A total of $50 \mu \mathrm{g}$ of protein extracted from cardiac and skeletal muscle tissue was separated by two-dimensional PAGE and the spot pattern visualized by silver staining as described (Hochstrasser et al. 1988). Twelve affected and 50 unaffected calves were analyzed.

\section{Protein identification}

The protein spots were cut from preparative gels, and digested with trypsin as described (Jensen et al. 1998). Digested protein spots were desalted using Zip tips, eluted with a $0.5-\mu \mathrm{L}$ matrix solution (15 g/L $\alpha$-cyano-4-hydroxycinnaminic acid in 70\% acetonitrile), and spotted directly on the MALDI-target. A Reflex-IV MALDI-TOF mass spectrometer (Bruker Daltonics) was used in a positive-ion reflector mode for peptide mass analyses, using the autolytic trypsin peaks of 842.11 and $2211.1 \mathrm{~m} / \mathrm{z}$ for internal calibration of spectra. Average deviation in mass accurracy was $50 \mathrm{ppm}$. Proteins were identified by peptide mass fingerprinting (PMF) using the database search program ProFound http:// prowl.rockefeller.edu/profound_bin/WebProFound.exe that searched the NCBI protein sequence database.

\section{Yeast methods and flow cytometry}

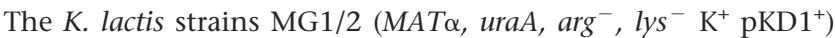
and $\mathrm{KL}_{3}\left(M A T \alpha\right.$, uraA, mnn2-2, $\left.\arg ^{-} \mathrm{K}^{+} \mathrm{pKD} 1^{+}\right)$used in this study and yeast transformation by electroporation has been described elsewhere (Abeijon et al. 1996). The vector pE4 (Guillen et al. 1998 ) was used for expression of bovine $S L C 35 A 3$ in yeast cells. $K$. lactis cells $\left(1.0 \mathrm{OD}_{600}\right)$ were labeled with FITC-conjugated WGA (Sigma) by washing the cells twice with labeling buffer $(50 \mathrm{mM}$ sodium phosphate, $150 \mathrm{mM} \mathrm{NaCl}, 1 \mathrm{mM} \mathrm{CaCl}_{2}, 0.5 \mathrm{mM} \mathrm{MgCl}$, $0.1 \mathrm{mM} \mathrm{MnCl}{ }_{2}$ at $\mathrm{pH}$ 7.0) followed by incubation with $15 \mu \mathrm{L}$ FITC-WGA $(2.5 \mathrm{mg} / \mathrm{mL})$ in $150 \mu \mathrm{L}$ labeling buffer for $1 \mathrm{~h}$ at $30^{\circ} \mathrm{C}$ with shaking. The cells were washed three times and finally resuspended in $1 \mathrm{~mL}$ of labeling buffer at room temperature. Immediately following FITC-WGA labeling, the cells were run through a Beckman Coulter Flow Cytometer.

\section{Acknowledgments}

We are grateful to C.B. Hirschberg and C. Abeijon for providing yeast strains and the $\mathrm{pE} 4$ vector, and P.J. de Jong for the bovine BAC library RPCI-42. The Danish Cattle Breeding Organizations supported this work.

\section{References}

Abeijon, C., Yanagisawa, K., Mandon, E.C., Hausler, A., Moremen, K., Hirschberg, C.B., and Robbins, P.W. 1993. Guanosine diphosphatase is required for protein and sphingolipid glycosylation in the Golgi lumen of Saccharomyces cerevisiae. J. Cell. Biol. 122: 307-323.

Abeijon, C., Robbins, P.W., and Hirschberg, C.B. 1996. Molecular cloning of the Golgi apparatus uridine diphosphate-N-acetylglucosamine transporter from Kluyveromyces lactis. Proc. Natl. Acad. Sci. 93: 5963-5968.

Abeijon, C., Mandon, E.C., and Hirschberg, C.B. 1997. Transporters of nucleotide sugars, nucleotide sulfate and ATP in the Golgi apparatus. Trends Biochem. Sci. 22: 203-207.

Agerholm, J.S., Bendixen, C., Andersen, O., and Arnbjerg, J. 2001. Complex vertebral malformation in holstein calves. J. Vet. Diagn. Invest. 13: 283-289.

Agerholm, J.S., Bendixen, C., Arnbjerg, J., and Andersen, O. 2004. Morphological variation of "complex vertebral malformation" in Holstein calves. J. Vet. Diagn. Invest. 16: 548-553.

Aulehla, A. and Herrmann, B.G. 2004. Segmentation in vertebrates: Clock and gradient finally joined. Genes \& Dev. 18: 2060-2067.

Aulehla, A., Wehrle, C., Brand-Saberi, B., Kemler, R., Gossler, A., Kanzler, B., and Herrmann, B.G. 2003. Wnt3a plays a major role in the segmentation clock controlling somitogenesis. Dev. Cell 4: $395-406$.

Berninsone, P., Miret, J.J., and Hirschberg, C.B. 1994. The Golgi guanosine diphosphatase is required for transport of GDP-mannose into the lumen of Saccharomyces cerevisiae Golgi vesicles. J. Biol. Chem. 269: 207-211.

Berninsone, P., Hwang, H.Y., Zemtseva, I., Horvitz, H.R., and Hirschberg, C.B. 2001. SQV-7, a protein involved in Caenorhabditis elegans epithelial invagination and early embryogenesis, transports UDP-glucuronic acid, UDP-N- acetylgalactosamine, and UDP-galactose. Proc. Natl. Acad. Sci. 98: 3738-3743.

Bruckner, K., Perez, L., Clausen, H., and Cohen, S. 2000. Glycosyltransferase activity of Fringe modulates Notch-Delta interactions. Nature 406: 411-415.

Bulik, D.A., Wei, G., Toyoda, H., Kinoshita-Toyoda, A., Waldrip, W.R., Esko, J.D., Robbins, P.W., and Selleck, S.B. 2000. sqv-3, -7, and -8, a set of genes affecting morphogenesis in Caenorhabditis elegans, encode enzymes required for glycosaminoglycan biosynthesis. Proc. Natl. Acad. Sci. 97: 10838-10843.

Bulman, M.P., Kusumi, K., Frayling, T.M., McKeown, C., Garrett, C., Lander, E.S., Krumlauf, R., Hattersley, A.T., Ellard, S., and Turnpenny, P.D. 2000. Mutations in the human delta homologue, DLL3, cause axial skeletal defects in spondylocostal dysostosis. Nat. Genet. 24: 438-441.

Cabral, C.M., Liu, Y., and Sifers, R.N. 2001. Dissecting glycoprotein quality control in the secretory pathway. Trends Biochem. Sci. 26: 619-624. 
Carrell, R.W. and Lomas, D.A. 2002. $\alpha 1$-antitrypsin deficiency-a model for conformational diseases. New Eng. J. Med. 346: 45-53.

Cartegni, L., Chew, S.L., and Krainer, A.R. 2002. Listening to silence and understanding nonsense: Exonic mutations that affect splicing. Nat. Rev. Genet. 3: 285-298.

Conlon, R.A., Reaume, A.G., and Rossant, J. 1995. Notch1 is required for the coordinate segmentation of somites. Development 121: $1533-1545$.

Crowther, D.C., Belorgey, D., Miranda, E., Kinghorn, K.J., Sharp, L.K., and Lomas, D.A. 2004. Practical genetics: $\alpha$-1-antitrypsin deficiency and the serpinopathies. Eur. J. Hum. Genet. 12: 167-172.

Dubrulle, J. and Pourquie, O. 2004. fgf8 mRNA decay establishes a gradient that couples axial elongation to patterning in the vertebrate embryo. Nature 427: 419-422.

Dubrulle, J., McGrew, M. J., and Pourquie, O. 2001. FGF signaling controls somite boundary position and regulates segmentation clock control of spatiotemporal Hox gene activation. Cell 106: 219-232.

Duncan, R.B.J., Carrig, C.B., Agerholm, J.S., and Bendixen, C. 2001. Complex vertebral malformation in a holstein calf: Report of a case in the USA. J. Vet. Diagn. Invest. 13: 333-336.

Evrard, Y.A., Lun, Y., Aulehla, A., Gan, L., and Johnson, R.L. 1998. lunatic fringe is an essential mediator of somite segmentation and patterning. Nature 394: 377-381.

Goto, S., Taniguchi, M., Muraoka, M., Toyoda, H., Sado, Y., Kawakita, M., and Hayashi, S. 2001. UDP-sugar transporter implicated in glycosylation and processing of Notch. Nat. Cell Biol. 3: 816-822.

Guillen, E., Abeijon, C., and Hirschberg, C.B. 1998. Mammalian Golgi apparatus UDP-N-acetylglucosamine transporter: Molecular cloning by phenotypic correction of a yeast mutant. Proc. Natl. Acad. Sci. 95: 7888-7892.

Hamada, Y., Kadokawa, Y., Okabe, M., Ikawa, M., Coleman, J.R., and Tsujimoto, Y. 1999. Mutation in ankyrin repeats of the mouse Notch2 gene induces early embryonic lethality. Development 126: $3415-3424$

Hicks, C., Johnston, S.H., diSibio, G., Collazo, A., Vogt, T.F., and Weinmaster, G. 2000. Fringe differentially modulates Jagged1 and Delta1 signalling through Notch1 and Notch2. Nat. Cell. Biol. 2: 515-520.

Hochstrasser, D.F, Harrington, M.G., Hochstrasser, A.C., Miller, M.J. and Merril, C.R. 1988. Methods for increasing the resolution of two-dimensional protein electrophoresis. Anal. Biochem. 173: $424-435$

Hoflich, J., Berninsone, P., Gobel, C., Gravato-Nobre, M.J., Libby, B.J. Darby, C., Politz, S.M., Hodgkin, J., Hirschberg, C.B., and Baumeister, R. 2004. Loss of srf-3-encoded nucleotide sugar transporter activity in Caenorhabditis elegans alters surface antigenicity and prevents bacterial adherence. J. Biol. Chem. 279: 30440-30448.

Hwang, H.Y. and Horvitz, H.R. 2002. The SQV-1 UDP-glucuronic acid decarboxylase and the SQV-7 nucleotide-sugar transporter may act in the Golgi apparatus to affect Caenorhabditis elegans vulval morphogenesis and embryonic development. Proc. Natl. Acad. Sci. 99: 14218-14223.

Ioffe, E. and Stanley, P. 1994. Mice lacking $\mathrm{N}$-acetylglucosaminyltransferase I activity die at mid-gestation, revealing an essential role for complex or hybrid N-linked carbohydrates. Proc. Natl. Acad. Sci. 91: 728-732.

Ishida, N. and Kawakita, M. 2004. Molecular physiology and pathology of the nucleotide sugar transporter family (SLC35). Pflugers Arch. 447: $768-775$

Ishida, N., Yoshioka, S., Chiba, Y., Takeuchi, M., and Kawakita, M. 1999. Molecular cloning and functional expression of the human Golgi UDP-N-acetylglucosamine transporter. J. Biochem. (Tokyo) 126: $68-77$.

Ishida, N., Kuba, T., Aoki, K., Miyatake, S., Kawakita, M., and Sanai, Y. 2005. Identification and characterization of human Golgi nucleotide sugar transporter SLC35D2, a novel member of the SLC35 nucleotide sugar transporter family. Genomics 85: 106-116.

Jensen, O.N., Larsen, M.R., and Roepstorff, P. 1998. Mass spectrometric identification and microcharacterization of proteins from electrophoretic gels: Strategies and applications. Proteins Suppl 2: 74-89.

Jiang, R., Lan, Y., Chapman, H.D., Shawber, C., Norton, C.R., Serreze, D.V., Weinmaster, G., and Gridley, T. 1998. Defects in limb, craniofacial, and thymic development in Jagged2 mutant mice. Genes \& Dev. 12: 1046-1057.

Kusumi, K., Sun, E.S., Kerrebrock, A.W., Bronson, R.T., Chi, D.C., Bulotsky, M.S., Spencer, J.B., Birren, B.W., Frankel, W.N., and Lander, E.S. 1998. The mouse pudgy mutation disrupts Delta homologue Dll3 and initiation of early somite boundaries. Nat. Genet. 19: $274-278$.
Lander, E.S. and Green, P. 1987. Construction of multilocus genetic linkage maps in humans. Proc. Natl. Acad. Sci. 84: 2363-2367.

Li, L., Krantz, I.D., Deng, Y., Genin, A., Banta, A.B., Collins, C.C., Qi, M., Trask, B.J., Kuo, W.L., Cochran, J., et al. 1997. Alagille syndrome is caused by mutations in human Jagged1, which encodes a ligand for Notch1. Nat. Genet. 16: 243-251.

Lopez-Avalos, M.D., Uccelletti, D., Abeijon, C., and Hirschberg, C.B. 2001. The UDPase activity of the Kluyveromyces lactis Golgi GDPase has a role in uridine nucleotide sugar transport into Golgi vesicles. Glycobiology 11: 413-422.

Lubke, T., Marquardt, T., Etzioni, A., Hartmann, E., von Figura, K., and Korner, C. 2001. Complementation cloning identifies CDG-IIc, a new type of congenital disorders of glycosylation, as a GDP-fucose transporter deficiency. Nat. Genet. 28: 73-76.

Luhn, K., Wild, M.K., Eckhardt, M., Gerardy-Schahn, R., and Vestweber, D. 2001. The gene defective in leukocyte adhesion deficiency II encodes a putative GDP-fucose transporter. Nat. Genet. 28: 69-72.

Marquardt, T. and Freeze, H. 2001. Congenital disorders of glycosylation: Glycosylation defects in man and biological models for their study. Biol. Chem. 382: 161-177.

Martinez-Duncker, I., Dupre, T., Piller, V., Piller, F., Candelier, J.J Trichet, C., Tchernia, G., Oriol, R., and Mollicone, R. 2005. Genetic complementation reveals a novel human congenital disorder of glycosylation of type II, due to inactivation of the Golgi CMP-sialic acid transporter. Blood 105: 2671-2676.

Mills, K., Mills, P.B., Clayton, P.T., Johnson, A.W., Whitehouse, D.B. and Winchester, B.G. 2001. Identification of $\alpha(1)$-antitrypsin variants in plasma with the use of proteomic technology. Clin. Chem. 47: 2012-2022.

Mills, K., Mills, P.B., Clayton, P.T., Mian, N., Johnson, A.W., and Winchester, B.G. 2003. The underglycosylation of plasma $\alpha$ 1-antitrypsin in congenital disorders of glycosylation type I is not random. Glycobiology 13: 73-85.

Moloney, D.J., Panin, V.M., Johnston, S.H., Chen, J., Shao, L., Wilson, R., Wang, Y., Stanley, P., Irvine, K.D., Haltiwanger, R.S., et al. 2000. Fringe is a glycosyltransferase that modifies Notch. Nature 406: $369-375$

Muraoka, M., Kawakita, M., and Ishida, N. 2001. Molecular characterization of human UDP-glucuronic acid/UDP-N-acetylgalactosamine transporter, a novel nucleotide sugar transporter with dual substrate specificity. FEBS Lett. 495: 87-93.

Nagahata, H., Oota, H., Nitanai, A., Oikawa, S., Higuchi, H., Nakade, T., Kurosawa, T., Morita, M., and Ogawa, H. 2002. Complex vertebral malformation in a stillborn Holstein calf in Japan. J. Vet. Med. Sci. 64: $1107-1112$.

Nielsen, U.S., Aamand, G.P., Andersen, O., Bendixen, C., Nielsen, V.H., and Agerholm, J.S. 2003. Effects of complex vertebral malformation on fertility traits in Holstein cattle. Livestock Prod. Sci. 79: 233-238.

Nishiwaki, K., Kubota, Y., Chigira, Y., Roy, S.K., Suzuki, M. Schvarzstein, M., Jigami, Y., Hisamoto, N., and Matsumoto, K. 2004. An NDPase links ADAM protease glycosylation with organ morphogenesis in C. elegans. Nat. Cell. Biol. 6: 31-37.

Oda, T., Elkahloun, A.G., Pike, B.L., Okajima, K., Krantz, I.D., Genin, A., Piccoli, D.A., Meltzer, P.S., Spinner, N.B., Collins, F.S., et al. 1997 Mutations in the human Jagged1 gene are responsible for Alagille syndrome. Nat. Genet. 16: 235-242.

Pourquie, O. 2003. The segmentation clock: Converting embryonic time into spatial pattern. Science 301: 328-330.

Princivalle, M. and de Agostini, A. 2002. Developmental roles of heparan sulfate proteoglycans: A comparative review in Drosophila, mouse and human. Int. J. Dev. Biol. 46: 267-278.

Revell, S. 2001. Complex vertebral malformation in a Holstein calf in the UK. Vet. Rec. 149: 659-660.

Rexroad, C.E., Schlapfer, J.S., Yang, Y., Harlizius, B., and Womack, J.E. 1999. A radiation hybrid map of bovine chromosome one. Anim. Genet. 30: 325-332.

Selva, E.M., Hong, K., Baeg, G.H., Beverley, S.M., Turco, S.J., Perrimon, N., and Hacker, U. 2001. Dual role of the fringe connection gene in both heparan sulphate and fringe-dependent signalling events. Nat. Cell Biol. 3: 809-815.

Shuster, D.E., Kehrli, M.E.J., Ackermann, M.R., and Gilbert, R.O. 1992. Identification and prevalence of a genetic defect that causes leukocyte adhesion deficiency in Holstein cattle. Proc. Natl. Acad. Sci. 89: 9225-9229.

Suda, T., Kamiyama, S., Suzuki, M., Kikuchi, N., Nakayama, K., Narimatsu, H., Jigami, Y., Aoki, T., and Nishihara, S. 2004. Molecular cloning and characterization of a human multisubstrate specific nucleotide-sugar transporter homologous to Drosophila fringe connection. J. Biol. Chem. 279: 26469-26474.

Toma, L., Pinhal, M.A., Dietrich, C.P., Nader, H.B., and Hirschberg, C.B.

\section{Genome Research}

www.genome.org 


\section{SLC35A3 required for axial skeleton formation}

1996. Transport of UDP-galactose into the Golgi lumen regulates the biosynthesis of proteoglycans. J. Biol. Chem. 271: 3897-3901.

Wang, Y., Tan, J., Sutton-Smith, M., Ditto, D., Panico, M., Campbell, R.M., Varki, N.M., Long, J.M., Jaeken, J., Levinson, S.R., et al. 2001. Modeling human congenital disorder of glycosylation type IIa in the mouse: Conservation of asparagine-linked glycan-dependent

functions in mammalian physiology and insights into disease pathogenesis. Glycobiology 11: 1051-1070.

Wouda, W., Visser, I.J., Borst, G.H., Vos, J.H., Zeeuwen, A.A., and Peperkamp, N.H. 2000. Developmental anomalies in aborted and stillborn calves in The Netherlands. Vet. Rec. 147: 612.

Zakany, J., Kmita, M., Alarcon, P., de la Pompa, J.L., and Duboule, D. 2001. Localized and transient transcription of Hox genes suggests a link between patterning and the segmentation clock. Cell 106: $207-217$

Zhang, N. and Gridley, T. 1998. Defects in somite formation in lunatic fringe-deficient mice. Nature 394: 374-377.

\section{Web site references}

http://prowl.rockefeller.edu/profound_bin/WebProFound.exe; ProFound tool for searching protein sequence databases.

http://www.phrap.org; PHRAP assembly engine.

Received January 12, 2005; accepted in revised form August 17, 2005. 


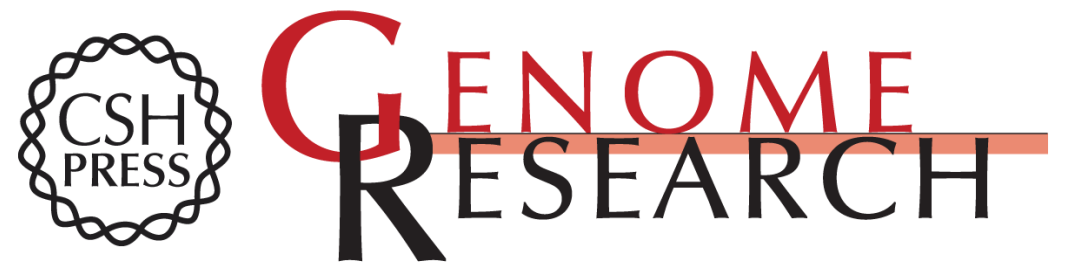

\section{A missense mutation in the bovine SLC35A3 gene, encoding a UDP- $N$-acetylglucosamine transporter, causes complex vertebral malformation}

Bo Thomsen, Per Horn, Frank Panitz, et al.

Genome Res. 2006 16: 97-105

Access the most recent version at doi:10.1101/gr.3690506

References This article cites 61 articles, 20 of which can be accessed free at:

http://genome.cshlp.org/content/16/1/97.full.html\#ref-list-1

Open Access Freely available online through the Genome Research Open Access option.

License

Email Alerting Receive free email alerts when new articles cite this article - sign up in the box at the Service top right corner of the article or click here.

\section{Affordable, Accurate Sequencing.}

